\title{
Interest of Surgical Treatment in the Management of Clavicle Fractures About 108 Cases
}

\author{
Rachid Maanouk ${ }^{*}$, S.Senhaji, A.Baroudi, M.Bensaka, Pr H.Abid, Pr M. El Idrissi, Pr A.Elibrahimi, Pr A.Elmrini
}

Department of Orthopedic, University Hospital Center HASSAN II Fez, 30000 fez, Morocco

DOI: $10.36347 /$ sjmcr.2020.v08i03.021

| Received: 16.02.2020 | Accepted: 23.02.2020 | Published: 18.03.2020

*Corresponding author: Rachid Maanouk

Abstract

Original Research Article

The treatment of clavicle fractures has been very recently subject to numerous studies which have questioned the traditional view that we had of this pathology with the thought that we obtain, in the vast majority of cases, an excellent functional result in case of surgical abstention. Indeed, these recent studies have shown that there is frequently a functional impact and a significant rate of non-union in certain types of fractures which could justify surgical treatment. If as a rule orthopedic treatment remains considered preponderant, surgical treatment keeps its indications however limited, but depends on several criteria including the type of fracture, the site, the associated lesions, the failure of the initial treatment and the occurrence of complications. Our work includes a series of 102 cases of clavicle fracture collected in the traumatology department B4 at the Hassan II University Hospital between January 2009 and June 2019. The objective is to analyze the clinical and radiological results of the surgical treatment of clavicle's fractures, as well as assess the functional outcome after surgical treatment.

Keywords: clavicle fracture, surgery, treatment.

Copyright @ 2020: This is an open-access article distributed under the terms of the Creative Commons Attribution license which permits unrestricted use, distribution, and reproduction in any medium for non-commercial use (NonCommercial, or CC-BY-NC) provided the original author and source are credited.

\section{INTRODUCTION}

Clavicle fractures are extremely common fractures, accounting for $2.6-5 \%$ of all fractures. They typically occur in children or young adult males. The most frequent clavicle fractures are those of the middle third ( 69 to $82 \%$ ), because of the anatomical features specific to this bone.

Orthopedic treatment plays a major role in the treatment of these fractures. However, in some cases, the risks of poor functional results, non-union, unsightly aesthetic results or the desire to resume activity more quickly encourage the offer of surgical treatment. There are many different operating techniques and the codification of indications is still often a school affair.

\section{Materials ANd Methods}

It is a retrospective descriptive and qualitative study. It concerned the analysis of 102 folder of patients admitted to the traumatology and orthopedic surgery department II of the HASSAN II University Hospital in Fez, treated surgically and followed in consultation for a clavicle fracture. This study covered a period of ten years from the beginning of the month January 2009 to June 2019.
Our goal is to analyze the clinical and radiological results of the surgical treatment of clavicle fractures, and to assess our functional results after surgical management.

\section{RESUlTS}

Our work includes a series of 102 cases of clavicle fracture treated surgically in the traumatology and orthopedic department II of the HASSAN II hospital in Fez, over a ten-year period from the beginning of January in the year 2009 to the month of June 2019 with an average decline of 26 months.

The average age was 36.9 years, with extremes of 19-72 years, with a male predominance $(83 \%)$. The right side was the most affected, with a percentage of $64 \%$. The direct was the most found in $75 \%$ of cases, indirect in $25 \%$ of cases. $60 \%$ of our patients were victims of AVP, followed by work accidents in $10 \%$ of cases. In our series the fracture was (according to ROBINSON's classification):

- Type 2B1: 64 cases $(62.7 \%)$

- $\quad$ Type 2B2: 25 cases $(24.5 \%)$

- $\quad$ Type 3B1: 13 cases $(12.8 \%)$ 
In our series 84 patients out of 102 presented a fracture of the middle third (82\%), and 18 cases presented a fracture of the external quarter $(18 \%)$, no fracture of the internal quarter been found.

We found 5 cases of acromioclavicular dislocation. 2 cases of skin opening. We have found no cases of pneumothorax or of hemothorax, and no cases of damage vascular and nerve associated. In $79.4 \%$ of the cases, the fracture was isolated, while in $20.6 \%$ of the cases, we had to deal with polytrauma or polyfractured patients with damage to neighboring elements:

- 2 cases of bilateral fracture of the clavicle with scapula fracture in 1 case.
- 5 cases of head trauma.

- 1 case of fracture of the base of the first right metacarpal (Bennet fracture).

- case of fracture of the contralateral leg.

- 1 case of fracture of the homolateral humeral palette.

- 1 case of femoral neck fracture.

- 1 case of dislocation of the elbow.

- 3 cases of fracture of the obturator frame.

- 4 cases of fracture of the lower extremity of the radius.

- 2 cases of fracture of the two bones of the forearm.

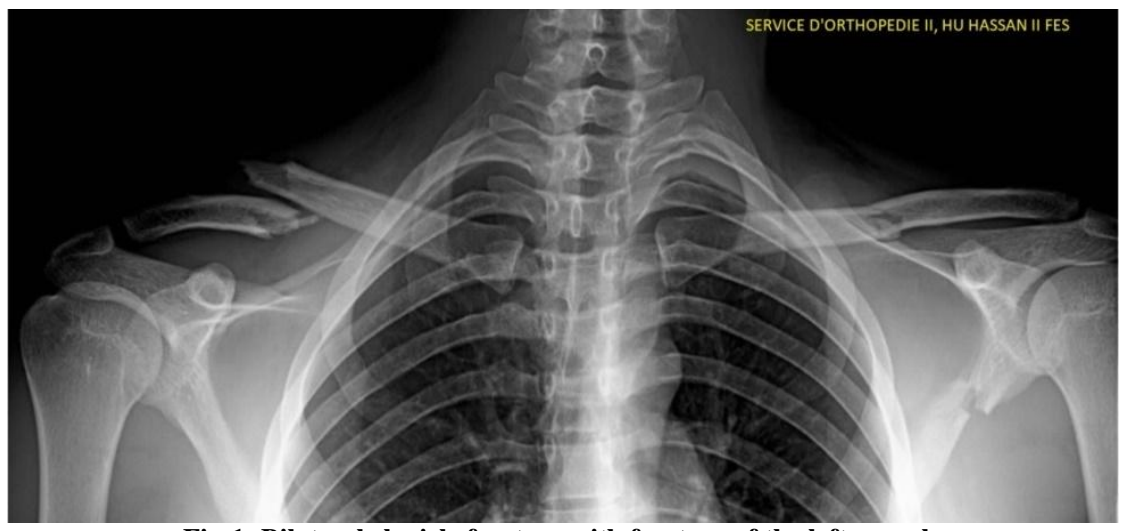

Fig-1: Bilateral clavicle fracture with fracture of the left scapula

Osteosynthesis was performed using a screw plate in 96 cases:

- $\quad$ S plate in 77 cases $(75.5 \%)$

- $\quad$ Third tube plate in 7 cases $(6.9 \%)$

- Hook plate in 12 cases $(17,6 \%)$

And by Intramedullary Nailing in 6 cases.

All of our patients were operated on in a halfseated position under general anesthesia, with an anterior approach by horizontal skin incision under the clavicle in all patients.

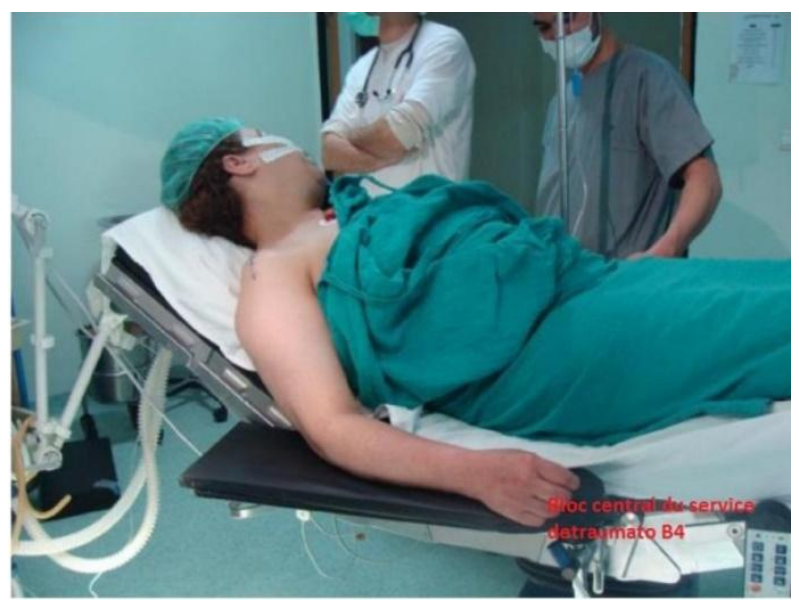

Fig-2: Installation of the patient in half-seated position

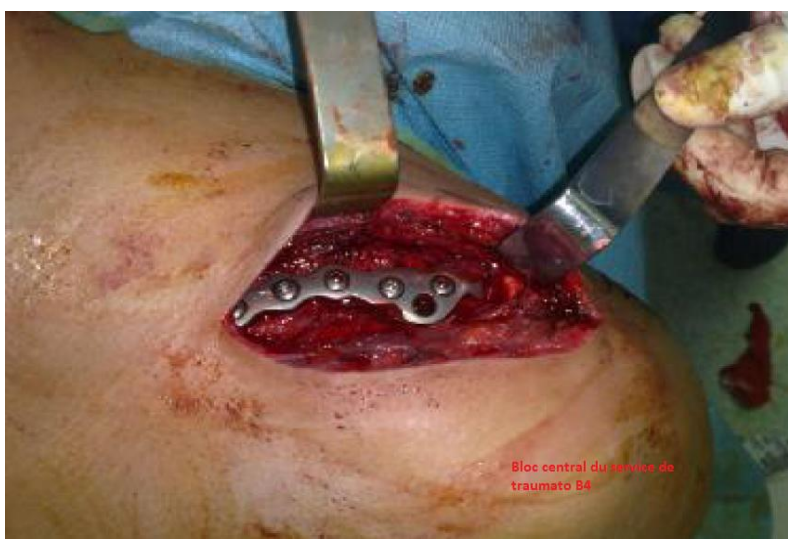

Fig-3: Fixing with a hook plate

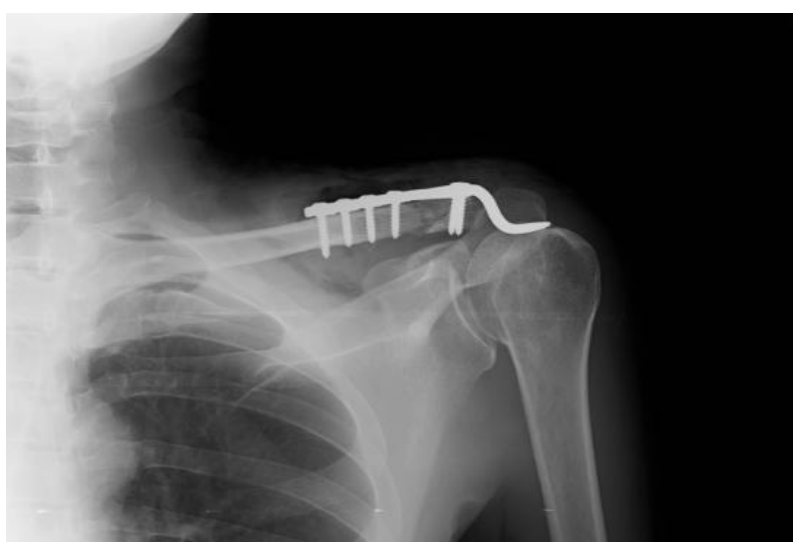

Fig-4: Post-operative control radiology 


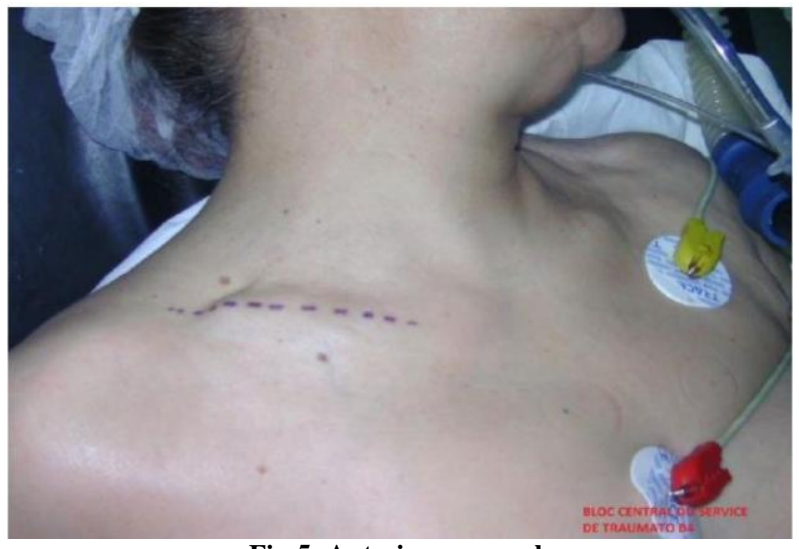

Fig-5: Anterior approach

Immobilization by simple sling or bandage elbow to the body for 14 days, were the means used in all the patients in our series.

Rehabilitation was started on $\mathrm{D}+1$ postoperatively in all patients.

The follow-up in consultation for a radioclinical control is ensured on D21, 2 months, 6 months then at 1 year.

The average duration of consolidation of our patients was 15 weeks.

7 patients presented post-operative complications with:

- 5 cases of sepsis on material

- 2 cases of exposure of osteosynthesis material

Our results were evaluated according to the clinical rating of the two shoulders described by CONSTANT [1] which is currently the file most recommended by the European society of shoulder and elbow surgery (SECEC). This rating is based on pain, daily activity level (Figure-8), painless active mobility and muscle strength.

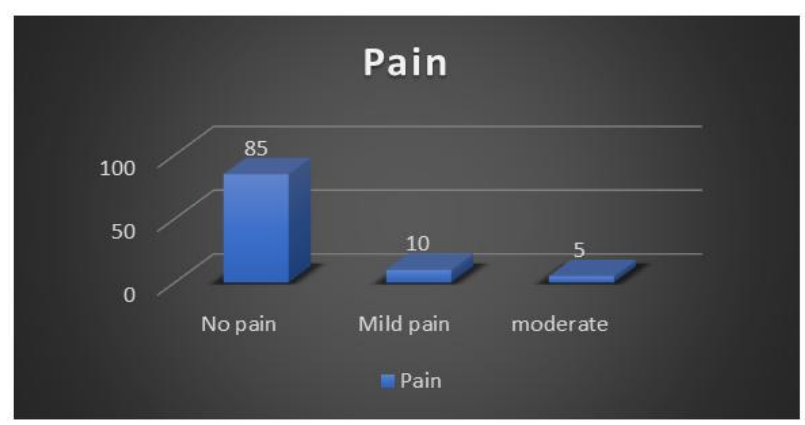

Fig-6: Results of postoperative shoulder pain According to the rating of CONSTANT

\section{muscle strength}

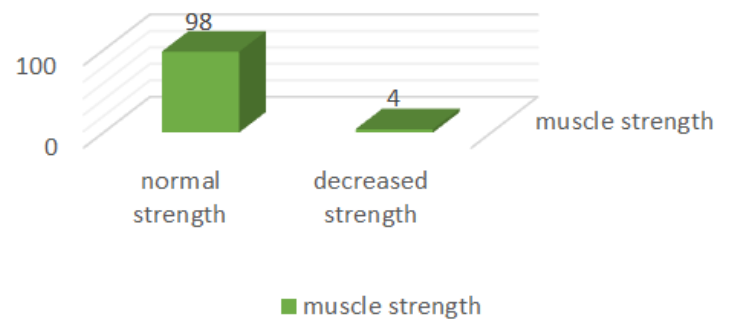

Fig-7: Postoperative results of muscle strength According to the rating of CONSTANT

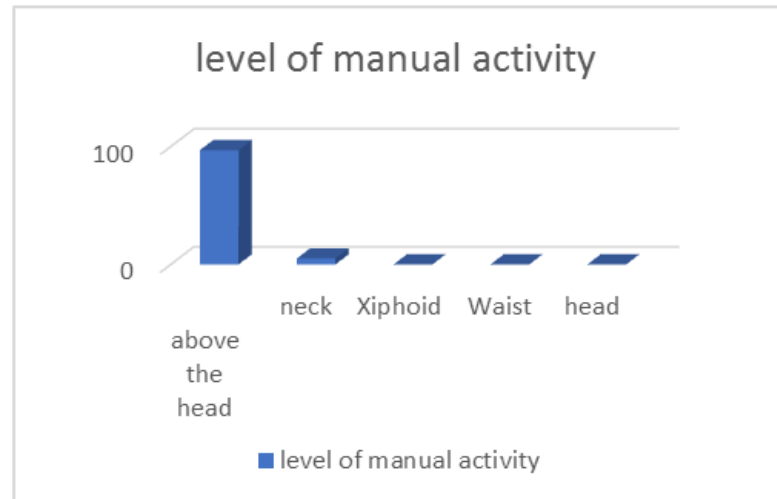

Fig-8: The results of the level of manual activity postoperatively According to the rating of CONSTANT

$85 \%$ had no pain, $10 \%$ complained of minimal pain and 5\% complained of moderate pain (Figure-6).

Muscle strength was normal in 98 cases. In the remaining 4 cases, muscle strength was decreased figure (Figure-7).

Regarding active mobility, the antepulsion was normal in all patients, reduction of the abduction to less than $90^{\circ}$ in 5 cases. 2 patients presented a limitation of the external rotation. For manual activity level, the hand is easily moved above the head in 97 cases. The hand does not exceed the neck in 5 cases.

The evaluation of the final result is objectified by the comparative analysis of the constant index on the operated side / versus the healthy side, namely the differential, which is in fact what is missing in the operated shoulder to be identical to that not operated (Table1 \& 2).

Table-1: Objective Qualitative evaluation result according constant as a function of the differential between the constant index of the operated side and the opposite Healthy side

\begin{tabular}{|l|l|l|l|l|}
\hline Deficit & \multicolumn{2}{|l|}{ Moderate } & Medium & Severe \\
\hline Differential (CI opposite side- CI operated side) & $\leq 10$ & $\leq 20$ & $\leq 30$ & $>30$ \\
\hline result & Excellent & Good & Medium & bad \\
\hline
\end{tabular}


Table-2: Functional results according to the CONSTAN rating

\begin{tabular}{|l|l|l|}
\hline Functional result & Number of cases & Percentage (\%) \\
\hline Excellent / good & $\mathbf{9 4}$ & $\mathbf{9 2 . 2 \%}$ \\
\hline Medium & $\mathbf{5}$ & $\mathbf{4 . 9 \%}$ \\
\hline Bad & $\mathbf{3}$ & $\mathbf{2 . 9 \%}$ \\
\hline
\end{tabular}

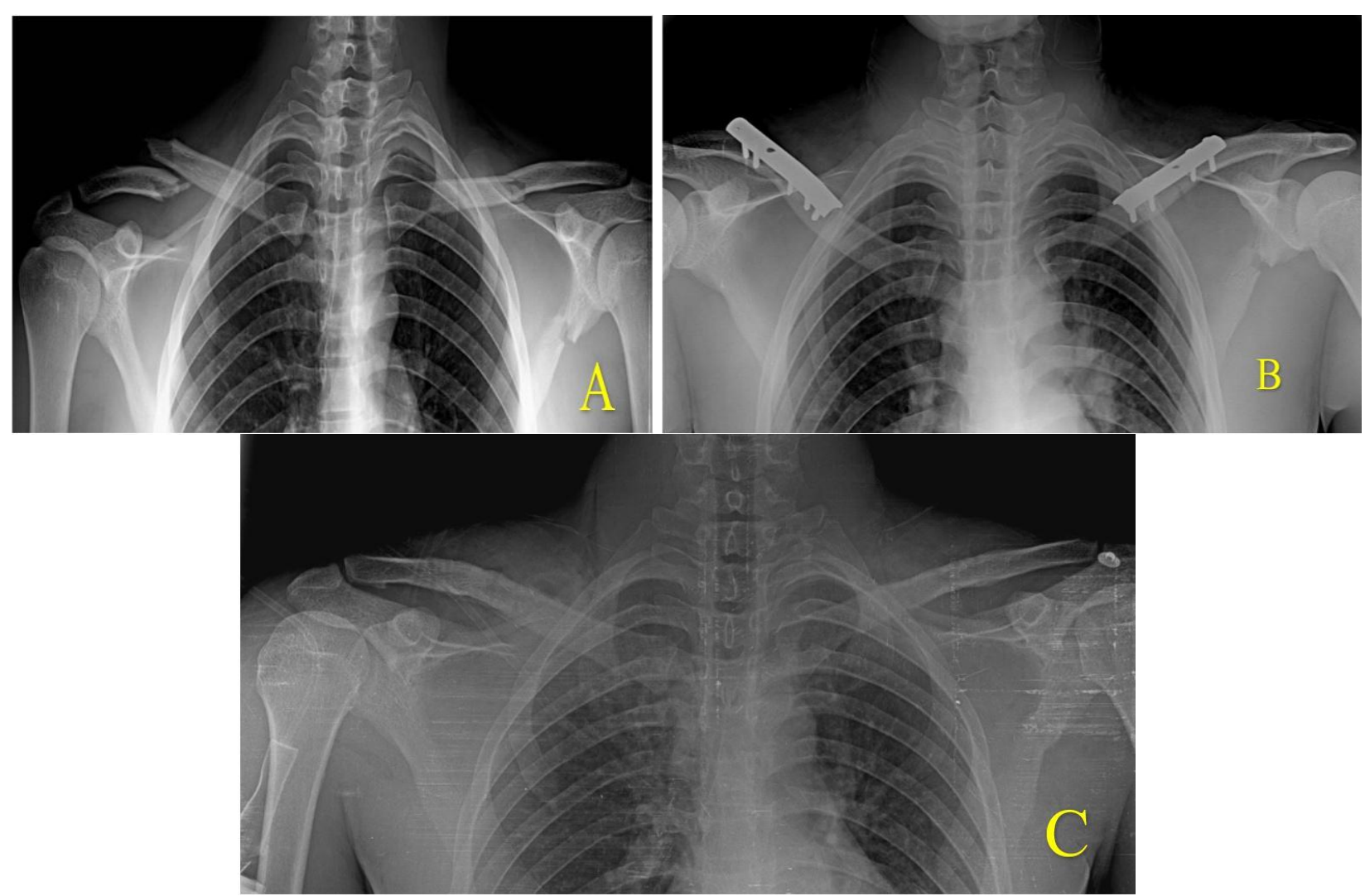

Fig-9: Bilateral clavicle fracture with fracture of the left scapula (A), surgically treated with two screw plate (B) consolidated and removal of osteosynthesis material after 1 year(C)

\section{DiSCUSSION}

The fracture of the clavicle is quite frequent, it represents according to F.Postacchini [2] $2.6 \%$ of all fractures and occurs especially in young and active adults [3]. There is a high frequency of sports accidents and accidents on the public highway [2-5]. The site most frequently found is the middle third fracture [6].

Although common, clavicle fracture does not mean an ordinary fracture due to the proximity of neurovascular elements and surrounding lung with are complications that may develop lifethreatening or functional [7-11].

The surgical management of clavicle fractures has been the subject of much debate. Although it is generally accepted that the treatment of non-displaced clavicle fractures is primarily functional, there remains an important debate on the type of immobilization [12].

The main advantage of orthopedic treatment [13-15] is its benignity, the absence of anesthetic and infectious risk is an argument often used. Not all authors agree on this generally recognized safety; the different techniques offered each have their own drawbacks: Lack of effectiveness in order sought, namely the stabilization of a reduction [16], skin lesions for the Elastoplast [17], and any pressure ulcers plasters with rings too tight and others.

The surgery aims are to restore bone integrity, which is the best way to avoid all of the functional consequences associated with hypertrophic callus and non-union, but it is unfortunately the price of additional risks [18].

A very large number of surgical techniques have been proposed for osteosynthesis of clavicle fractures. The plate fixation is the surgical technique reference, Molded plates "S" are those recommended by McKee [19], other plates can be used as the plate $1 / 3$ tube, plate DCP or LCP 3.5 [20], the plate Mennen [21].

When there is a loss of substance, a spongy graft complementary to contemporary osteosynthesis is strongly recommended. 
There are traditional indications for which all surgeons agree on the need for surgical stabilization. Doctor Sartorius in his thesis entitled: "the interest of operating a good number of clavicle fracture" made it well [22]. In 1983, they offered the following indications:

- $\quad$ Large displacement fractures if the reduction is not stable

- Comminuted fractures with an intermediate fragment, all the more so as that or these are perpendicular to the focal point of the fracture,

- Fractures in athletes [13]

- Open fractures

- Very comminuted fractures.

Surgical treatment allows earlier mobilization allowing a marked improvement in functional results and a reduction in consolidation delays, vicious calluses and nonunion. As shown in the series by Altamini and Mckee [23] highlighting a clear functional deference between plate osteosynthesis versus orthopedic treatment of clavicle fractures.

Lazarides and Zafiropoulos [24] have shown that patient dissatisfaction with their outcome after orthopedic treatment of a mediocavicular displaced fracture increases with the value of the shortening (from $18 \mathrm{~mm}$ in men and $14 \mathrm{~mm}$ in women) and can reach up to $25 \%$ of patients.

The complications generally reported after the use of osteosynthesis by plaque are essentially infection, plaque fracture, hypertrophic or painful scars, mobilization of the screws, non- union, iterative fractures after removal of plaque and vascular lesions per -opératoires [25-28].

\section{CONCLUSION}

At the end of this work and after seeing the literature, it is clear that the problem of indication of orthopedic or surgical treatment, fractures of the clavicle still remains. However, when surgical treatment is necessary, the indication of the surgical technique to be used should depend on the different characteristics of the fracture. Osteosynthesis using a screw plate remains, according to the literature, the most effective method. Non-union remains the most frequently reported complication in the literature, thus considered to result from an error in surgical indication or the choice of surgical technique. In all cases, an X-ray assessment adapted to a precise analysis and a good appreciation of the terrain are essential to better manage of this type of fractures.

\section{REFERENCES}

1. Constant CR, Murley AH. A clinical method of functional assessment of the shoulder. Clinical orthopaedics and related research. 1987 $\operatorname{Jan}(214): 160-4$.
2. Postacchini F, Gumina S, De Santis P, Albo F. Epidemiology of clavicle fractures. Journal of Shoulder and Elbow Surgery. 2002 Sep $1 ; 11(5): 452-6$.

3. Robinson CM. Fractures of the clavicle in the adult: epidemiology and classification. The Journal of bone and joint surgery. British volume. 1998 May;80(3):476-84.

4. Singh R, Rambani R, Kanakaris N, Giannoudis PV. A 2-year experience, management and outcome of 200 clavicle fractures. Injury. 2012 Feb 1;43(2):159-63.

5. Lindenmaier HL, Kuner EH, Becker B. Osteosynthesis of the clavicle. Indications, surgical technique, results. Der Chirurg; Zeitschrift fur alle Gebiete der operativen Medizen. 1991 May;62(5):409-13.

6. De Giorgi S, Notarnicola A, Tafuri S, Solarino G, Moretti L, Moretti B. Conservative treatment of fractures of the clavicle. BMC research notes. 2011 Dec;4(1):333.

7. Williams GR, Naranja J, Klimkiewicz J, Karduna A, Iannotti JP, Ramsey M. The floating shoulder: a biomechanical basis for classification and management. JBJS. 2001 Aug 1;83(8):1182-7.

8. Barbier O, Malghem J, Delaere O, Vande Berg B, Rombouts JJ. Injury to the brachial plexus by a fragment of bone after fracture of the clavicle. The Journal of bone and joint surgery. British volume. 1997 Jul;79(4):534-6.

9. Della Santa D, Narakas A, Bonnard C. Late lesions of the brachial plexus after fracture of the clavicle. InAnnales de Chirurgie de la Main et du Membre Supérieur 1991 Jan 1 (Vol. 10, No. 6, pp. 531-540). Elsevier Masson.

10. Favard L, Berhouet J, Bacle G. Traumatisme de l'épaule et de la ceinture scapulaire. EMC. 2009.

11. Raviraja A, Chandrashekar CM, Roshan SD, Srinivas JV. Subclavian artery and vein injury following clavicle fracture due to blunt trauma. Injury Extra. 2009 Jan 1;40(2):36-8.

12. Lester CW. The Treatment of fracures of the Clavicle. Ann Surg.1929, 89:600-606.

13. Basamania. Fractures of the clavicle InRockwood Jr C, Matsen III F, Wirth M, Lippitt S (ed) 2004. The Shoulder ed 3. Saunders, Philadelphia, 455520.

14. Forgue. Fractures de la clavicule. Traité de Thérapeutique Chirurgical Masson, 1992; 463 468.

15. Lahbadi S. Huit plâtré pour fracture du tiers moyen de la clavicule. Technique orthopédique illustrée, traumatologie des membres. 1990.

16. Lindenmaier HL, Kuner EH, Becker B. Osteosynthesis of the clavicle. Indications, surgical technique, results. Der Chirurg; Zeitschrift fur alle Gebiete der operativen Medizen. 1991 May;62(5):409-13.

17. Webber MC, Haines JF. The treatment of lateral clavicle fractures. Injury. 2000 Apr 1;31(3):175-9. 
18. Pidhorz L. Traumatisme de la ceinture scapulaire. Encycl Méd Chir, 1998; 14-085-A-10: 20.

19. Altamimi SA, McKee MD. Nonoperative treatment compared with plate fixation of displaced midshaft clavicular fractures: Surgical technique. JBJS. 2008 Mar 1;90(Supplement_2_Part_1):1-8.

20. Bouillet B, Moreel P, Descamps S. Prise en charge des fractures récentes de la clavicule. Journal de traumatologie du sport. 2009 Mar 1;26(1):24-31.

21. Russo R, Visconti V, Lorini S, Lombardi LV. Displaced comminuted midshaft clavicle fractures: use of Mennen plate fixation system. Journal of Trauma and Acute Care Surgery. 2007 Oct 1;63(4):951-4.

22. Sartorius C. De l'intérêt à synthéser par plaque" tiers de tube" bon nombre de fractures de la clavicule: à propos de 414 observations: 383 fractures fraîches, 24 pseudarthroses, 7 cals vicieux. Thèse de med Grenoble, 1983.

23. Altamimi SA, McKee MD. Canadian Orthopaedic Trauma Society. Nonoperative treatment compared with plate fixation of displaced midshaft clavicular fractures. Surgical technique. J Bone Joint Surg Am. 2008;90(suppl 2):1-8.

24. Lazarides S, Zafiropoulos G. Conservative treatment of fractures at the middle third of the clavicle: the relevance of shortening and clinical outcome. Journal of shoulder and elbow surgery. 2006 Mar 1;15(2):191-4.

25. Kashf K. Les Fractures De La Clavicule. Maîtrise Orthopédique, 2009; 184.

26. Charles S Neer II. 5 Fractures of the distal third of the clavicle. Clinical Orthopaedics and Related Research (1976-2007). 1968 May 1;58:43-50.

27. Mall JW, Jacobi CA, Philipp AW, Peter FJ. Surgical treatment of fractures of the distal clavicle with polydioxanone suture tension band wiring: an alternative osteosynthesis. Journal of orthopaedic science. 2002 Sep 1;7(5):535-7.

28. Gazielly. Fractures de la clavicule. L'épaule au quotidien, 1999, 114-116. 